\title{
The Impact of Staple Line Reinforcement Utilization on Bleeding and Leak Rates Following Sleeve Gastrectomy for Severe Obesity: a Propensity and Case-Control Matched Analysis
}

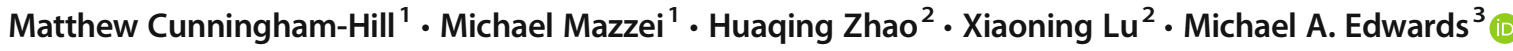

Published online: 15 April 2019

(C) Springer Science+Business Media, LLC, part of Springer Nature 2019

\begin{abstract}
Introduction Staple line reinforcement (SLR) is a commonly used technique during laparoscopic sleeve gastrectomy (SG) for severe obesity. There remains controversy over the potential benefit or risk associated with SLR. There are currently no consensus recommendations about SLR use. Its use is surgeon-dependent and remains controversial.

Study Aim To determine the impact of staple line reinforcement on staple line leak and bleeding rates after sleeve gastrectomy. Methods Using the Metabolic and Bariatric Surgery Quality Improvement Program Participant User File (MBSQIP-PUF) database, we identified patients who had a SG in 2015 and 2016. SLR utilization status was used to create two cohorts. An unmatched cohort analysis was performed, and the outcomes were compared. A propensity score and case-control matched cohort analysis were then performed, and the outcomes were compared. Statistical analysis was performed with SPSS and SAS. Results Of the 189,173 SG cases identified, SLR utilization was noted in 127,521 (67.4\%). In the unmatched analysis, bleeding and reoperation were significantly higher in the cohort without SLR utilization. In both propensity score and case-control matched analysis, bleeding and reoperation remained significantly higher in the cohort without SLR utilization. There was no difference in mortality and staple line leak rates between the cohorts.

Conclusions SLR significantly reduces bleeding and reoperation rates following SG and has no deleterious impact on staple line leak rate. While further prospective studies factoring in the SLR method and staple characteristics are needed, this large database analysis supports the use of routine SLR during SG to reduce the risk of perioperative bleeding and reoperation.
\end{abstract}

Keywords Sleeve gastrectomy $\cdot$ Staple line reinforcement $\cdot$ Leak rate $\cdot$ Bleeding rate

\section{Introduction}

Worldwide obesity has continued to increase in prevalence over the last several decades [1]. Metabolic and bariatric surgery (MBS) has been proven to be an effective, sustainable, and safe method of weight loss [2-5]. Since the 1960s, bariatric surgery as a treatment option for severe obesity has

Michael A. Edwards

edwards.michael@mayo.edu; maeedw@gmail.com

1 Department of Surgery, Division of Minimally Invasive and Bariatric Surgery, Temple University Hospital, Parkinson Pavilion Suite 410, 3401, North Broad Street, Philadelphia, PA 19140, USA

2 Division of Biostatistics, Lewis Katz School of Medicine at Temple University, Philadelphia, PA, USA

3 Department of Surgery, Division of General Surgery, Mayo Clinic, 4500 San Pablo Rd S, Jacksonville, FL 3224, USA continued to evolve in technique and the types of operations offered. Over the last 8 years, we have seen a dramatic shift in metabolic and bariatric operations performed, from primarily laparoscopic gastric bypass (LRYGB) and laparoscopic adjustable gastric band (LAGB) to LRYGB and laparoscopic sleeve gastrectomy (SG) $[2,5]$. Over this time period, SG has become the most commonly performed MBS procedure in the US, representing over $50 \%$ of cases [5-7].

The safety and efficacy of SG for severe obesity are wellestablished [2-5, 8-12]. Perioperative and intermediate outcomes are reportedly similar to those of gastric bypass $[4,13$, 14]. Percent excess weight loss (\%EWL) for SG at 1,4 , and 5 years is reported in recent literature as $82.0 \pm 18.8,72.8 \pm$ 22.6 , and $60.3 \pm 28.9 \%$, respectively [11]. While some studies have reported similar weight loss, others have reported higher short- and intermediate-term weight loss with LRYGB compared to SG $[2,4,14]$. Remission of obesity-related comorbid conditions following $\mathrm{SG}$ is also reportedly similar to that of 
gastric bypass $[2,3,15,16]$. SG has a reported 30-day morbidity and mortality of 5.6 and $0.06-0.11 \%$, respectively, which is comparable to the reported morbidity $(5.9 \%)$ and mortality $(0.13-0.14 \%)$ rates following gastric bypass $[17$, $18]$.

As SG continues to grow in the proportion of metabolic and bariatric operations performed, there remain concerns about the type and incidence of complications that will need management perioperatively or during the intermediate- and long-term postoperative follow-up periods. Staple line leak and bleeding following metabolic and bariatric surgery remains a challenging problem for bariatric surgeons and is associated with increased patient morbidity, mortality, hospital length of stay, and cost [5, 17]. Even though staple line leak following SG is a rare complication, it is particularly challenging to manage and may require months to resolve. The rate of staple line leak after SG is reportedly $0-8 \%$ and is similar to the reported anastomotic leak rates after LRYGB (0.1-8.3\%) [17-21]. Bleeding following SG is reportedly $0.75-4.94 \%$, compared to 1.6-3.3\% following LRYGB [5, 22-25].

While staple line reinforcement (SLR) techniques vary [19], they are commonly used during sleeve gastrectomy and are generally thought to reduce the risk of staple line leak and bleeding [9]. Berger et al. [5] estimated that $80 \%$ of surgeons use some form of SLR during sleeve gastrectomy. In our recent review of the 2015 and 2106 Metabolic and Bariatric Surgery Accreditation and Quality Improvement Program Participant User File (MBSAQIP-PUF), 67\% of the surgeons reported using SLR during SG. Even though this is a commonly used technique, there are currently no consensus guidelines on the optimal technique for SLR during SG, and whether it should or should not be routinely used remains a point of discussion and controversy [9]. Studies have reported a reduction, an increase, and no impact on bleeding and staple line leak rates when staple line reinforcement is used during SG $[5,8,10,12,19-21,24,26,27]$.

Using the largest, national, de-identified bariatric clinical dataset, we sought to determine the impact of using SLR on bleeding and staple line leak complications following sleeve gastrectomy by comparing matched cohorts to reduce confounding variables.

\section{Methods}

\section{Data Source and Inclusion Criteria}

The Metabolic and Bariatric Surgery Accreditation and Quality Improvement Program Participant User File (MBSAQIP-PUF) collects prospective risk-adjusted, clinical data based on standardized definitions of preoperative, intraoperative, and postoperative variables that are specific for metabolic and bariatric surgery $[5,10]$. Data is abstracted by certified clinical reviewers (MBSCRs) at participating institutions, as previously described [5, 28-30]. For the purposes of this study, the MBSAQIP database was queried to identify all patients who underwent a laparoscopic sleeve gastrectomy between January 1, 2015, and December 31, 2016, using current procedural terminology (CPT) code 43775 . Cases with missing data, patients $<18$ years old, revision/conversion cases, and cases in which initial surgical approach was not robotic-assisted or conventional laparoscopic were excluded from the analysis. A flow diagram of inclusion and exclusion criteria is depicted in Fig. 1. To account for possible confounding variables that may impact outcome measures, propensity score and case-control matching were performed.

\section{Data Collection and Outcome Measures}

Descriptive statistics were collected and compared between cohorts, including patient demographics, such as age, gender, race/ethnicity, and preoperative BMI closest to surgery, health status variable, such as ASA classification, and preexisting comorbid conditions, including hypertension requiring medication, hyperlipidemia, myocardial infarction, renal insufficiency, chronic renal disease requiring dialysis, vein thrombosis requiring therapy, history of pulmonary emboli, diabetes mellitus, current smoking within 1 year, obstructive sleep apnea, chronic obstructive lung disease, oxygen dependence, chronic steroid/immunosuppressant, limited ambulation status, partial functional dependence, total functional dependence, venous stasis, and previous bariatric or foregut surgery. Thirty-one outcome measures were assessed, including operative length, hospital length of stay, conversion from initial procedure, discharge status, 30-day intensive care unit (ICU) admission, reoperation, readmission, intervention, and mortality, drain present at 30 days, death likely related to bariatric surgery, renal failure, progressive renal insufficiency, cardiopulmonary resuscitation (CPR), coma $>24 \mathrm{~h}$, stroke, myocardial infarction, deep venous thrombosis (DVT) requiring therapy, pulmonary emboli (PE), anticoagulation for presumed/ confirmed DVT/PE, transfusion, pneumonia (PNA), unplanned intubation, on ventilator $>48 \mathrm{~h}$, urinary tract infection (UTI), sepsis, septic shock, superficial surgical site infection (SSI), deep SSI, organ space SSI, and peripheral nerve injury. Eight aggregate complications were also compared, including aggregate leak, bleeding, renal, cardiovascular, and pulmonary complications, venous thromboembolic events, aggregate SSI, and other infection. Aggregate complication methodology is defined in Appendix Table 8, as previously described by Berger et al. [5]. Outcome measures were analyzed and reported for the entire patient cohort, as well as for unmatched and matched cohorts. Our primary outcome measures were bleeding and staple line leak rates. 


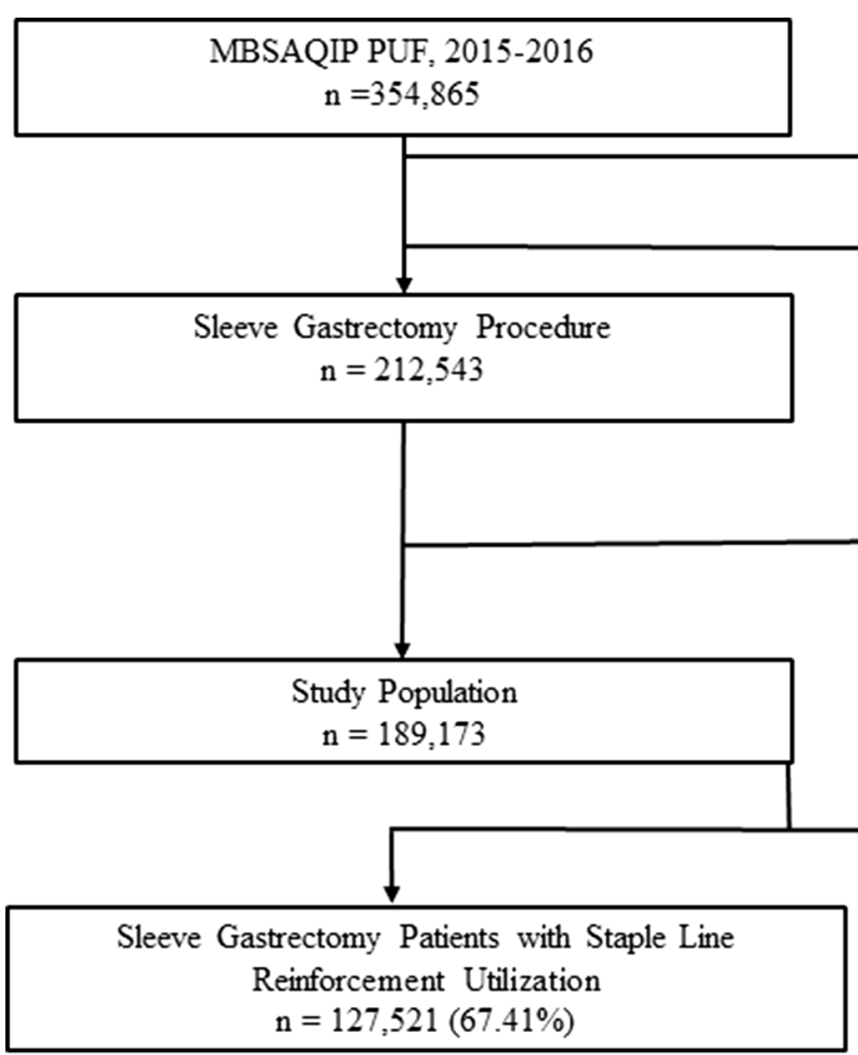

Fig. 1 Flow diagram of case selection. MBSAQIP, Metabolic and Bariatric Surgery Accreditation and Quality Improvement Program; PUF, participant use data file; LRYGB, laparoscopic Roux-en-Y gastric

\section{Matched Analysis}

To determine the impact of staple line reinforcement utilization on perioperative outcomes, while controlling for confounding variables, matched analyses were performed on cases (no SLR utilization) and controls (SLR utilization) using both propensity score and case-control matching techniques. For propensity score matching, a logistic regression model was generated, in which SLR utilization status was regressed on baseline characteristics that were thought to serve as potential confounders; from this, a propensity score was assigned to each subject based on the probability of staple line utilization given other covariates. 1:1 matching of case and control subjects with similar propensity scores was then used to generate new cohorts hypothesized to be balanced on important potentially confounding baseline characteristics. For propensity score calculation, candidate variables for regression consisted of all available demographic characteristics and preoperative comorbidities. For case-control matching, a $1: 1,1: 3$, and 1:5 matching ratio was performed. To ensure a more representative sample of age and bougie size distribution in the matched cohorts, age [<35 (9\%); 35-45 (31.7\%); 45-55 (245.4\%); 55-65
Non-Weight Loss Primary Procedure

$\mathrm{n}=32,493$

Other Bariatric Procedure $\mathrm{n}=109,829$

LRYGB: $89,187(25.2 \%)$

LAGB: $7857(2.2 \%)$

BPD-DS: $2852(0.8 \%)$

Missing Data, Age $<18$, Revision procedure, Non robotic or laparoscopic procedure $\mathrm{n}=23,370$

\section{Sleeve Gastrectomy Patients without Staple Line Reinforcement Utilization $\mathrm{n}=61,652(32.59 \%)$}

bypass; LAGB, laparoscopic adjustable gastric banding; BPD-DS, biliopancreatic diversion with duodenal switch

$(12.3 \%)$; and $>65(2.5 \%)]$ and bougie size $[<40 \mathrm{~F}$ or $\geq$ 40F] categories were created, and cohorts were matched on these categories. Cases and controls were matched by patient demographics, as well as by preoperative comorbidities and operative characteristics that were significantly different in unmatched analysis of the SLR and no SLR cohorts. Case-control matched cohorts were further stratified by operation duration.

\section{Statistical Analysis}

Univariate analyses of the entire cohort and unmatched cohorts were performed using Pearson's $\chi^{2}$ test for categorical variables, independent sample $t$ tests for normally distributed continuous variables, and Mann-Whitney $U$ tests for skewed continuous variables. A similar univariate analysis was repeated on the propensity score and casecontrol matched cohorts. Continuous variables were reported as mean \pm standard deviation and categorical variables as percentage. All statistical analysis was performed with SPSS version 25 (IBM Corporation, Armonk, NY) or SAS version 9.4 (SAS Institute, Cary, NC). A $p$ value $<0.05$ was considered statistically significant. 


\section{Results}

\section{Baseline Characteristics}

A total of 189,173 sleeve gastrectomy cases were identified in the 2015 and 2016 MBSAQIP-PUF database and included in our analysis. Staple line reinforcement was used in 127,521 (67.4\%). Patient demographics and disease prevalence of the entire cohort and unmatched cohorts are detailed in Table 1. Overall, $78.9 \%$ was female and the mean age (years) was $44.4 \pm 12$. There were 18.4 and $12.3 \%$ Black and Hispanic patients, respectively. The mean preoperative weight and body mass index (BMI) were $278.1 \pm 59.4 \mathrm{lb}$ and $45.2 \pm 8 \mathrm{~kg} / \mathrm{m}^{2}$, respectively. Surgical approach included $93.2 \%$ conventional laparoscopic and $6.8 \%$ robotic-assisted cases. The mean bougie size used was $34.9 \pm 8.8$ French. On average, $\mathrm{SG}$ was initiated $4.6 \pm 2.1 \mathrm{~cm}$ proximal to the pylorus.

Unmatched cohort analysis compared 61,652 cases without SLR utilization to 127,521 controls with SLR utilization. At baseline, there were significant differences in preoperative patient and operative characteristics between the two cohorts. In the SLR utilization cohort, the patients were significantly younger (44.3 years vs. 44.7 years, $p<0.0001)$. Staple line reinforcement was used in a higher proportion of female $(79.1 \%$ vs. $78.8 \%, p=0.2)$ and Black (18.6\% vs. $18 \%, p$ 0.0007) patients. The SLR utilization cohort also had a significantly higher prevalence of gastroesophageal reflux $(28.8 \%$ vs. $27.6 \%, p<0.0001)$ and preoperative inferior vena cava (IVC) filter placement $(0.9 \%$ vs. $0.7 \%, p<0.0001)$. In those without SLR utilization, there was a significantly higher prevalence of diabetes mellitus $(23.5 \%$ vs. $22.9 \%, p=0.01)$, insulin use $(6.8 \%$ vs. $6.6 \%, p=0.04)$, myocardial infarction $(1.3 \%$ vs. $1.2 \%, p 0.0003$ ), hyperlipidemia ( $23 \%$ vs. $22.6 \%, p=$ $0.03)$, percutaneous coronary intervention $(2.2 \%$ vs. $2.0 \%, p=0.006)$, hyperlipidemia ( $23 \%$ vs. $22.6 \%, p=$ $0.03)$, chronic obstructive pulmonary disease $(1.8 \%$ vs. $1.6 \%, p=0.002)$, oxygen dependence $(0.8 \%$ vs. $0.5 \%$, $p<0.0001)$, venous thromboembolic events $(1.6 \%$ vs. $1.4 \%, p<0.0001)$, partial functional dependence $(0.7 \%$ vs. $0.6 \%, p=0.001)$, total functional dependence $(0.7 \%$ vs. $0.3 \%, p<0.0001)$, and venous stasis $(1.1 \%$ vs. $0.9 \%$, $p=0.004)$. The use of SLR varied with initial surgical approach $(p=0.002)$ in this unmatched comparison. SLR was used more frequently in conventional laparoscopic cases $(93.3 \%$ vs. $92.9 \%)$ and less often in robotic-assisted cases $(6.8 \%$ vs. $7.1 \%)$. In the SLR utilization cohort, the mean bougie size used was larger ( 35 vs. $34.7, p<0.0001)$ and SG was initiated closer to the pylorus $(2.0 \mathrm{~cm}$ vs. $2.4 \mathrm{~cm}$, $p=0.02$ ). All other patient and operative characteristics were similar between the two cohorts.

\section{Perioperative Outcomes}

Perioperative outcomes for the entire cohort are outlined in Table 2, highlighting the overall safety of sleeve gastrectomy in the treatment of severe obesity. Overall, operative length and postoperative length of stay were $74.2 \mathrm{~min}$ and 1.6 days. Conversion to another approach was $0.09 \%$. The 30 -day outcomes were low, including 30 -day mortality of $0.07 \%$, of which $0.03 \%$ was related to the bariatric operation. Overall bleeding, leak, and venous thromboembolic event (VTE) rates were $0.27,0.24$, and $0.60 \%$, respectively. Perioperative outcomes of the unmatched SLR and no SLR cohorts are also detailed in Table 2. SLR utilization was associated with longer operative duration (minutes) $(75.1$ vs. $72.5, p<0.0001)$ and postoperative length of stay (HLOS) (days) (1.7 vs. 1.6, $p<0.0001$ ). At baseline, SLR utilization was also associated with a significantly lower rate of postoperative deep SSI $(0.02 \%$ vs. $0.03 \%, p=0.03)$, pneumonia $(0.1 \%$ vs. $0.2 \%$, $p=0.03)$, sepsis $(0.06 \%$ vs. $0.1 \%, p=0.001)$, transfusion within $72 \mathrm{~h}(0.4 \%$ vs $0.6 \%, p<0.0001)$, and aggregate bleeding $(0.2 \%$ vs $0.3 \%, p=0.0005)$. All other complications were similar between the two cohorts at baseline but generally lower with staple line reinforcement use.

The 30-day adverse outcomes were generally equal to or lower in the SLR utilization cohort, including a significantly lower reoperation rate $(0.8 \%$ vs. $1.0 \%, p=0.0004)$. There was no mortality difference $(0.07 \%$ vs. $0.08 \%, p=0.8)$. Aggregate complications, representing the rates of certain systems-based complications associated with the bariatric surgery leading to readmission, re-intervention, or reoperation, are also detailed in Table 2. All the aggregate complications were similar between study cohorts, except for a higher bleeding rate without SLR use $(p=0.0005)$.

\section{Perioperative Outcomes in Propensity Score Match Analysis}

The extent of the propensity score matching success and the resulting cohort demographics are detailed in Table 3. Propensity score matching resulted in matched cohorts consisting of 128,150 patients, with 63,075 in each cohort. The cohorts were statistically equal in overall health status and comorbid conditions. Following propensity score matching, BMI (45.3 vs. 45.2, $p=0.02$ ) remained higher in the cohort with SLR utilization. Perioperative, 30-day, and aggregate outcomes following propensity score match are outlined in Table 4. After the propensity match, operative length (75.1 $\mathrm{min}$ vs. $72.6 \mathrm{~min}, p=0.001)$ and postoperative length of stay (1.7 days vs. 1.6 days, $p=0.001)$ remained significantly higher in those with SLR utilization. There was no mortality difference $(p=0.4)$. Reoperation $(0.8 \%$ vs. $1 \%$, $\mathrm{RR}=0.8, p<0.001)$, transfusion $(0.4 \%$ vs. $0.6 \%, \mathrm{RR}=0.7$, $p=0.001)$, deep SSI $(0.01 \%$ vs. $0.04 \%, p=0.002)$, sepsis 
Table 1 Demographics and disease prevalence in unmatched staple line reinforcement cohorts

All cases $[n=189,173] \quad$ (+) SLR utilization $[n=127,521] \quad(-)$ SLR utilization $[n=61,652] \quad p$ value

Continuous variables, mean \pm standard deviation

\begin{tabular}{|c|c|c|c|c|}
\hline Age (years) & $44.4 \pm 12$ & $44.3 \pm 12$ & $44.7 \pm 12$ & $<0.0001$ \\
\hline Weight closest to surgery (lb) & $278.1 \pm 59.4$ & $277.90 \pm 59.3$ & $278.6 \pm 59.6$ & 0.02 \\
\hline BMI closest to surgery $\left(\mathrm{kg} / \mathrm{m}^{2}\right)$ & $45.2 \pm 8$ & $45.15 \pm 8$ & $45.2 \pm 8.0$ & 0.06 \\
\hline \multicolumn{5}{|l|}{ Categorical variables, $n(\%)$} \\
\hline Gender (male) & $39,780(21.0)$ & $26,708(20.9)$ & $13,072(21.2)$ & 0.2 \\
\hline Gender (female) & $149,393(79)$ & $100,813(79.1)$ & $48,580(78.8)$ & 0.2 \\
\hline Race (White) & $120,089(63.5)$ & $81,072(63.6)$ & $39,017(63.3)$ & 0.2 \\
\hline Race (Black) & $34,798(18.4)$ & $23,724(18.6)$ & $11,074(18)$ & 0.0007 \\
\hline Ethnicity (Hispanic) & $23,266(12.3)$ & $15,616(12.3)$ & $7650(12.4)$ & 0.3 \\
\hline ASA class & & & & $<0.0001$ \\
\hline 1 & $720(0.4)$ & $474(0.4)$ & $246(0.4)$ & \\
\hline 2 & $47,919(25.3)$ & $32,180(25.2)$ & $15,739(25.5)$ & \\
\hline 3 & $134,311(71.0)$ & $91,424(71.7)$ & $42,887(69.6)$ & \\
\hline 4 & $6211(3.3)$ & $3433(2.7)$ & $2778(4.5)$ & \\
\hline 5 & $12(0.01)$ & $10(0.01)$ & $2(0.003)$ & \\
\hline \multicolumn{5}{|l|}{ Preoperative disease prevalence } \\
\hline GERD & $53,724(28.4)$ & $36,716(28.8)$ & $17,008(27.6)$ & $<0.0001$ \\
\hline History of MI & $2298(1.2)$ & $1469(1.2)$ & $829(1.3)$ & 0.0003 \\
\hline History of PCI & $3830(2.0)$ & $2502(2.0)$ & $1328(2.2)$ & 0.006 \\
\hline History cardiac surgery & $2200(1.2)$ & $1513(1.2)$ & $687(1.1)$ & 0.2 \\
\hline Hypertension & $89,354(47.2)$ & $60,177(47.2)$ & $29,177(47.3)$ & 0.6 \\
\hline Hyperlipidemia & $42,937(22.7)$ & $28,762(22.6)$ & $14,175(23.0)$ & 0.03 \\
\hline Diabetes mellitus & $43,697(23.1)$ & $29,234(22.9)$ & $14,463(23.5)$ & 0.01 \\
\hline Insulin use & $12,613(6.7)$ & $8400(6.6)$ & $4213(6.8)$ & 0.04 \\
\hline COPD & $3192(1.7)$ & $2070(1.6)$ & $1122(1.8)$ & 0.002 \\
\hline OSA & $67,723(35.8)$ & $45,681(35.8)$ & $22,042(35.8)$ & 0.8 \\
\hline Oxygen dependent & $1167(0.6)$ & $682(0.5)$ & $485(0.8)$ & $<0.0001$ \\
\hline Smoker & $16,936(9)$ & $11,487(9.0)$ & $5449(8.8)$ & 0.2 \\
\hline CKD & $1228(0.7)$ & $802(0.6)$ & $426(0.7)$ & 0.1 \\
\hline Dialysis & $592(0.3)$ & $400(0.3)$ & $192(0.3)$ & 0.9 \\
\hline VTE requiring therapy & $2796(1.5)$ & $1783(1.4)$ & $1013(1.6)$ & $<0.0001$ \\
\hline History of PE & $2066(1.1)$ & $1404(1.1)$ & $662(1.1)$ & 0.6 \\
\hline IVC filter & $1608(0.9)$ & $1200(0.9)$ & $408(0.7)$ & $<0.0001$ \\
\hline Anticoagulation & $4456(2.4)$ & $2941(2.3)$ & $1515(2.5)$ & 0.04 \\
\hline Venous stasis & $1808(1.0)$ & $1161(0.9)$ & $647(1.1)$ & 0.004 \\
\hline Chronic steroid & $3282(1.7)$ & $2217(1.7)$ & $1065(1.7)$ & 0.9 \\
\hline Limited ambulation status & 3104 (1.6) & 2092 (1.6) & $1012(1.6)$ & 0.2 \\
\hline Partial functional dependence & $1205(0.6)$ & $759(0.6)$ & $446(0.7)$ & 0.001 \\
\hline Total functional dependence & $124(0.1)$ & $62(0.3)$ & $62(0.7)$ & $<0.0001$ \\
\hline
\end{tabular}

SLR, staple line reinforcement; $n$, sample size; lb, pound; $\mathrm{kg}$, kilogram; $\mathrm{m}^{2}$, meter square; ASA, American Society of Anesthesiologist; GERD, gastroesophageal reflux; MI, myocardial infarction; PCI, percutaneous coronary intervention; COPD, chronic obstructive pulmonary disease; OSA, obstructive sleep apnea; CKD, chronic kidney disease; PE, pulmonary emboli; IVC, inferior vena cava

$(0.1 \%$ vs. $0.1 \%, \mathrm{RR}=0.6, p=0.01)$, aggregate bleeding $(0.3 \%$ vs. $0.3 \%, \mathrm{RR}=0.7, p=0.004)$ and aggregate SSI $(0.4 \%$ vs. $0.5 \%, p=0.02)$ remained significantly lower in the SLR utilization cohort. Leak rate was similar between cohorts $(p=0.5)$.

\section{Perioperative Outcomes in Case-Control Match Analysis}

Cohorts with and without SLR utilization were matched with a 1:1, 1:3, and 1:5 ratio. Cases and controls were matched on 
Table 2 Outcomes in unmatched cohorts

\begin{tabular}{|c|c|c|c|c|}
\hline & All cases $[n=189,173]$ & (+) SLR utilization $[n=127,521]$ & (-) SLR utilization $[n=61,652]$ & $p$ value \\
\hline Operative duration (minutes)* & $74.2 \pm 37.2$ & $75.1 \pm 36.9$ & $72.5 \pm 37.9$ & $<0.0001$ \\
\hline Hospital LOS (days)* & $1.6 \pm 1.5$ & $1.7 \pm 1.6$ & $1.6 \pm 1.3$ & $<0.0001$ \\
\hline Approach converted, $n(\%)$ & $179(0.09)$ & $114(0.09)$ & $65(0.1)$ & 0.3 \\
\hline \multicolumn{5}{|c|}{ 30-Day outcomes and perioperative complications $n(\%)$} \\
\hline Follow-up & $180,644(95.5)$ & $122,068(95.7)$ & $58,576(95.01)$ & $<0.0001$ \\
\hline ICU admission & $991(0.5)$ & $651(0.5)$ & $340(0.6)$ & 0.2 \\
\hline Reoperation & $1623(0.7)$ & $1027(0.8)$ & $596(1.0)$ & 0.0004 \\
\hline Readmission & $6066(3.2)$ & $4071(3.2)$ & $1995(3.2)$ & 0.6 \\
\hline Re-intervention & $1815(1.0)$ & $1218(1.0)$ & $597(1.0)$ & 0.8 \\
\hline Mortality & $140(0.07)$ & $93(0.07)$ & $47(0.08)$ & 0.8 \\
\hline Death related & $66(0.03)$ & $43(0.03)$ & $23(0.04)$ & 0.7 \\
\hline Transfusion & $928(0.5)$ & $557(0.4)$ & $371(0.6)$ & $<0.0001$ \\
\hline Acute renal failure & $108(0.06)$ & $67(0.05)$ & $41(0.07)$ & 0.9 \\
\hline Progressive renal failure & $107(0.06)$ & $73(0.06)$ & $34(0.06)$ & 0.2 \\
\hline CPR & $59(0.03)$ & $38(0.03)$ & $21(0.03)$ & 0.6 \\
\hline Coma $>24 \mathrm{~h}$ & $7(0.0)$ & $4(0.0)$ & $3(0.0)$ & 0.6 \\
\hline Stroke & $23(0.01)$ & $19(0.01)$ & $4(0.01)$ & 0.1 \\
\hline Myocardial infarction & $54(0.03)$ & $34(0.03)$ & $20(0.03)$ & 0.5 \\
\hline On ventilator $>48 \mathrm{~h}$ & $96(0.05)$ & $54(0.04)$ & $42(0.07)$ & 0.06 \\
\hline Organ space SSI & $299(0.2)$ & $202(0.2)$ & $97(0.2)$ & 0.9 \\
\hline Deep incisional SSI & $44(0.02)$ & $23(0.02)$ & $21(0.03)$ & 0.03 \\
\hline Superficial SSI & $428(0.2)$ & $275(0.2)$ & $153(0.3)$ & 0.2 \\
\hline Pneumonia & $254(0.1)$ & $155(0.1)$ & $99(0.2)$ & 0.03 \\
\hline Unplanned intubation & $222(0.1)$ & $147(0.1)$ & $75(0.1)$ & 0.7 \\
\hline DVT requiring therapy & $330(0.2)$ & $214(0.2$ & $116(0.2)$ & 0.3 \\
\hline Pulmonary emboli & $172(0.09)$ & $109(0.09)$ & $63(0.1)$ & 0.3 \\
\hline Anticoagulation for DVT/PE & $823(0.4)$ & $563(0.4)$ & $260(0.4)$ & 0.5 \\
\hline Postoperative sepsis & $150(0.08)$ & $82(0.06)$ & $68(0.1)$ & 0.001 \\
\hline Postoperative septic shock & $59(0.03)$ & $37(0.03)$ & $22(0.04)$ & 0.4 \\
\hline Postoperative UTI & $544(0.3)$ & $378(0.3)$ & $166(0.3)$ & 0.3 \\
\hline Nerve injury & $3(0.0)$ & $2(0.0)$ & $1(0.002)$ & 0.9 \\
\hline \multicolumn{5}{|l|}{ Aggregate complications, $n(\%)$} \\
\hline Bleeding & $504(0.3)$ & $303(0.2)$ & $201(0.3)$ & 0.0005 \\
\hline Leak & $463(0.2)$ & $316(0.3)$ & $147(0.2)$ & 0.7 \\
\hline Cardiovascular & $150(0.08)$ & $104(0.08)$ & $46(0.07)$ & 0.6 \\
\hline Pulmonary & $666(0.4)$ & $442(0.4)$ & $224(0.4)$ & 0.6 \\
\hline Renal & $236(0.1)$ & $152(0.1)$ & $84(0.1)$ & 0.3 \\
\hline VTE & $1135(0.6)$ & $775(0.6)$ & $360(0.6)$ & 0.5 \\
\hline SSI & $862(0.5)$ & $559(0.4)$ & $303(0.5)$ & 0.1 \\
\hline Other infection & $1149(0.6)$ & $763(0.6)$ & $386(0.6)$ & 0.5 \\
\hline Total infection & $1819(1.0)$ & $1196(1.0)$ & $623(1.0)$ & 0.1 \\
\hline
\end{tabular}

SLR, staple line reinforcement; $n$, sample size; *, data missing in 164; LOS, postoperative length of stay; ICU, intensive care unit; CPR, cardiopulmonary resuscitation; SSI, surgical site infection; DVT, deep vein thrombosis; PE, pulmonary emboli; UTI, urinary tract infection; VTE, venous thromboembolic event $P$ values that were statistically significant were bolded

patient demographics (age, BMI, gender, and race), as well as preoperative comorbidities and operative characteristics that were found to be significantly different in unmatched comparison of the two cohorts. Matching covariates are detailed in
Table 5. A 1:1 case-control matching identified 107,050 patients, with 53,525 in each cohort. Postoperative length of stay was significantly higher in the SLR cohort (1.7 days vs. 1.5 days, $p<0.0001)$. The 30 -day outcomes were similar 
Table 3 Patient characteristics following 1:1 propensity score matching

(+) SLR utilization $[n=64,075$

(-) SLR utilization $[n=64,075]$

RR

$p$ value

Continuous variables, mean \pm standard deviation

\begin{tabular}{|c|c|c|c|c|}
\hline Age (year) & $44.6 \pm 12.1$ & $44.6 \pm 12.0$ & 1.00 & 0.4 \\
\hline ASA classification & $2.8 \pm 0.5$ & $2.8 \pm 0.5$ & 1.00 & 0.3 \\
\hline Weight closest (pound) & $278.9 \pm 60.3$ & $278.5 \pm 59.5$ & 1.00 & 0.01 \\
\hline BMI closest $\left(\mathrm{kg} / \mathrm{m}^{2}\right)$ & $45.3 \pm 8.0$ & $45.2 \pm 8.1$ & 1.01 & 0.02 \\
\hline \multicolumn{5}{|l|}{ Categorical variables (\%) } \\
\hline Gender (male) & 21.5 & 21.2 & 1.01 & 0.3 \\
\hline Race (White) & 63.4 & 63.3 & 1.00 & 0.6 \\
\hline Race (Black) & 50.0 & 50.0 & 1.00 & 0.8 \\
\hline Ethnicity (Hispanic) & 12.2 & 12.2 & 0.99 & 0.9 \\
\hline Race (Asian) & 0.5 & 0.5 & 0.96 & 0.6 \\
\hline \multicolumn{5}{|l|}{ Preoperative disease prevalence (\%) } \\
\hline GERD & 27.7 & 27.6 & 1.00 & 0.6 \\
\hline History of MI & 1.3 & 1.3 & 1.01 & 0.9 \\
\hline History of PCI & 2.1 & 2.1 & 0.98 & 0.7 \\
\hline History of previous cardiac surgery & 1.1 & 1.1 & 0.99 & 0.9 \\
\hline Hypertension requiring medication & 47.4 & 47.2 & 1.00 & 0.4 \\
\hline Hyperlipidemia & 23.1 & 23.0 & 1.01 & 0.6 \\
\hline Diabetes mellitus & 23.6 & 23.4 & 1.00 & 0.6 \\
\hline Insulin & 6.8 & 6.8 & 1.00 & 0.9 \\
\hline COPD & 1.8 & 1.8 & 0.99 & 0.8 \\
\hline Obstructive sleep apnea & 36.0 & 35.6 & 1.01 & 0.1 \\
\hline Oxygen dependent & 0.8 & 0.8 & 1.01 & 0.9 \\
\hline Current smoker within 1 year & 8.8 & 8.7 & 1.02 & 0.4 \\
\hline Renal insufficiency & 0.7 & 0.7 & 1.00 & 0.9 \\
\hline Dialysis & 0.3 & 0.3 & 1.00 & 0.9 \\
\hline Vein thrombosis requiring therapy & 1.6 & 1.7 & 0.98 & 0.7 \\
\hline History of PE & 1.0 & 1.1 & 0.94 & 0.2 \\
\hline IVC filter & 0.6 & 0.7 & 0.99 & 0.9 \\
\hline Therapeutic anticoagulation & 2.5 & 2.5 & 1.02 & 0.5 \\
\hline Venous stasis & 1.0 & 1.0 & 1.00 & 0.9 \\
\hline Steroid/immunosuppressant use & 1.7 & 1.7 & 1.02 & 0.7 \\
\hline Limited ambulation status & 1.6 & 1.6 & 1.00 & 0.9 \\
\hline Partial functional dependence & 0.7 & 0.7 & 0.98 & 0.7 \\
\hline Total functional dependence & 0.5 & 0.5 & 0.97 & 0.7 \\
\hline Previous surgery & 1.6 & 1.6 & 0.98 & 0.6 \\
\hline
\end{tabular}

SLR, staple line reinforcement; $n$, sample size; RR, relative risk; ASA, American Society of Anesthesiologist; BMI, body mass index; GERD, gastroesophageal reflux; MI, myocardial infarction; PCI, percutaneous coronary intervention; COPD, chronic obstructive pulmonary disease; PE, pulmonary emboli; IVC, inferior vena cava

$P$ values that were statistically significant were bolded

between cohorts, except for a significantly lower reoperation rate $(0.7 \%$ vs. $0.9 \%, p=0.0006)$ in the cohort with SLR utilization. Postoperative transfusion $(0.4 \%$ vs. $0.5 \%, p 0.009)$ and aggregate bleeding $(0.2 \%$ vs. $0.3 \%, p=0.02)$ were also significantly lower in the SLR utilization cohort. Mortality rate $(p=$ $0.7)$, leak rate $(p=0.6)$, and all other outcome measures were similar between cases and controls (Table 6). There were no outcome differences when a 1:1, 1:3, and 1:5 matching ratio was used in our case-control analysis; however, the sample sizes were smaller for the 1:3 and 1:5 matching ratio cohorts and were excluded from our results. Several differences were noted between the propensity score and case-control matched analysis. Propensity score matching showed a significantly lower rate of postoperative sepsis, deep incision SSI, and aggregate SSI in the cohort with SLR use $(p=0.01,0.002,0.02)$; however, these outcome variables were similar between study 
Table 4 Outcomes following 1:1 propensity score matching

\begin{tabular}{|c|c|c|c|c|}
\hline & (+) SLR utilization $[n=64,075]$ & (-) SLR utilization $[n=64,075]$ & $\mathrm{RR}$ & $p$ value \\
\hline Operative length (minutes) & $75.1 \pm 37.6$ & $72.6 \pm 37.8$ & 1.0 & 0.001 \\
\hline Postoperative LOS (days) & $1.7 \pm 1.9$ & $1.6 \pm 1.3$ & 1.1 & 0.001 \\
\hline Approach converted (\%) & 0.1 & 0.1 & 1.0 & 0.9 \\
\hline \multicolumn{5}{|c|}{ 30-Day outcomes and perioperative complication (\%) } \\
\hline 30-Day ICU admission & 0.5 & 0.6 & 0.9 & 0.09 \\
\hline Reoperation & 0.8 & 1.0 & 0.8 & $<0.001$ \\
\hline Readmission & 3.2 & 3.2 & 1.0 & 0.5 \\
\hline Re-intervention & 0.9 & 1.0 & 1.0 & 0.5 \\
\hline 30-Day mortality & 0.1 & 0.1 & 0.8 & 0.4 \\
\hline Transfusion within $72 \mathrm{~h}$ & 0.4 & 0.6 & 0.7 & $<0.001$ \\
\hline Acute renal failure & 0.1 & 0.1 & 0.8 & 0.3 \\
\hline Progressive renal insufficiency & 0.1 & 0.1 & 1.1 & 0.8 \\
\hline Cardiopulmonary resuscitation & 0.03 & 0.04 & 0.9 & 0.6 \\
\hline Coma $>24 \mathrm{~h}$ & 0.00 & 0.00 & 0.3 & 0.3 \\
\hline Stroke & 0.02 & 0.01 & 2.0 & 0.2 \\
\hline Myocardial infarction & 0.02 & 0.03 & 0.7 & 0.3 \\
\hline On ventilator $>48 \mathrm{~h}$ & 0.04 & 0.1 & 0.6 & 0.06 \\
\hline Organ space SSI & 0.2 & 0.2 & 1.0 & 0.9 \\
\hline Deep SSI & 0.01 & 0.04 & 0.3 & 0.002 \\
\hline Superficial SSI & 0.2 & 0.3 & 0.8 & 0.05 \\
\hline Pneumonia & 0.1 & 0.2 & 0.8 & 0.2 \\
\hline Unplanned intubation & 0.1 & 0.1 & 1.1 & 0.6 \\
\hline DVT requiring therapy & 0.2 & 0.2 & 0.8 & 0.2 \\
\hline Pulmonary embolism & 0.1 & 0.1 & 0.9 & 0.5 \\
\hline Anticoagulation for DVT/PE & 0.4 & 0.4 & 1.0 & 0.6 \\
\hline Postoperative sepsis & 0.1 & 0.1 & 0.6 & 0.01 \\
\hline Postoperative septic shock & 0.03 & 0.04 & 0.8 & 0.5 \\
\hline Postoperative UTI & 0.3 & 0.3 & 1.0 & 0.8 \\
\hline \multicolumn{5}{|l|}{ Aggregate complications (\%) } \\
\hline Bleeding & 0.3 & 0.3 & 0.7 & 0.004 \\
\hline Leak & 0.2 & 0.2 & 0.9 & 0.5 \\
\hline Cardiovascular & 0.1 & 0.1 & 0.9 & 0.7 \\
\hline VTE & 0.4 & 0.4 & 1.0 & 0.9 \\
\hline Renal & 0.1 & 0.1 & 0.9 & 0.3 \\
\hline Pulmonary & 0.6 & 0.6 & 1.0 & 0.6 \\
\hline SSI & 0.4 & 0.5 & 0.8 & 0.02 \\
\hline Other infection & 0.6 & 0.6 & 0.9 & 0.3 \\
\hline
\end{tabular}

SLR, staple line reinforcement; $n$, sample size; RR, relative risk; SD, standard deviation; LOS, length of stay; ICU, intensive care unit; CPR, cardiopulmonary resuscitation; CVA, cerebrovascular accident; SSI, surgical site infection; DVT, deep venous thrombosis; PE, pulmonary emboli

$P$ values that were statistically significant were bolded

cohorts $(0.06 \%$ vs. $0.09 \%, p=0.1 ; 0.02 \%$ vs. $0.03 \%, p=0.2$; and $0.4 \%$ vs $0.5 \%, p=0.6$ ) following case-control matched analysis.

In order to limit surgeon experience as a potential confounder, 1:1 case-control matching was repeated and stratified by operation duration. In this matched analysis, 83,608 patients were identified and compared, with 41,804 in each cohort. The outcomes are reported in Table 7. Mean postoperative length of stay remained significantly higher in the SLR utilization cohort (1.6 days vs. 1.5 days, $p<0.0001)$. The 30 -day reoperation $(0.7 \%$ vs $0.9 \%, p 0.0004)$ and ICU admission ( $0.3 \%$ vs. $0.4 \%, p=0.04)$ were significantly lower in the SLR utilization cohort. Transfusion within $72 \mathrm{~h}(0.4 \%$ vs $0.5 \%, p=$ $0.0001)$ and aggregate bleeding $(0.2 \%$ vs. $0.3 \%, p=0.004)$ also remained significantly lower in this cohort. Pneumonia $(0.07 \%$ vs $0.1 \%, p=0.006$ ) and aggregate pulmonary complications 
Table 5 Descriptive statistics after case-control matching

Variable

$p$ value*

Continuous variables

Age

Preoperative weight closest to surgery

Preoperative BM closest to surgery

Categorical variables

Race/ethnicity

Sex

ASA classification

Preoperative comorbidities

History of myocardial infarction

Previous PCI/PTCA

Hyperlipidemia

Vein thrombosis requiring therapy

Therapeutic anticoagulation for presumed VTE

IVC filter

Partial functional dependence

Total functional dependence

Venous stasis

Diabetes mellitus

Insulin use

Current smoker within 1 year

Chronic obstructive pulmonary disease

Oxygen dependent

Surgical approach

Bougie size

Distance from pylorus

$\begin{array}{ll}\text { AGE } & 1 \\ \text { PREOP_WEIGHTCLOSEST } & 0.6 \\ \text { PREOP_BMICLOSEST } & 0.9 \\ & \\ \text { RACE } & 1 \\ \text { GENDER } & 1 \\ \text { ASA_CLASS } & 1 \\ & \\ \text { PMH_MI } & 1 \\ \text { PMH_PTC } & 1 \\ \text { PMH_HLD } & 1 \\ \text { PMH_DVT } & 1 \\ \text { PMH_ANTICOAG } & 1 \\ \text { PMH_IVCFILTER } & 1 \\ \text { PHM_PARTIALDEP } & 1 \\ \text { PMH_FULLDEP } & 1 \\ \text { PMH_VENSTASIS } & 1 \\ \text { PMH_DIAB } & 1 \\ \text { INSULIN } & 1 \\ \text { PMH_SMOKER } & 1 \\ \text { PMH_COPD } & 1 \\ \text { PMH_OXYGEN } & 1 \\ \text { OR_INITIALAPPROACH } & 1 \\ \text { OR_BOUGIESIZE } & 1 \\ \text { OR_PYLORUS } & 1 \\ & \\ & \end{array}$

${ }^{*} p$ value after case-control matching

BMI, body mass index; ASA, American Society of Anesthesiologist; PTC, percutaneous coronary intervention; PTCA, percutaneous coronary angioplasty; VTE, venous thromboembolic event; IVC, inferior vena cava; PMH, past medical history

$(0.2 \%$ vs $0.3 \%, p=0.02)$, which were similar in the earlier case-control matched analysis, became significantly lower in the SLR utilization cohort once operative length was included as a matching covariate. All other 30-day outcome measures, complications, and aggregate complications were similar between the two cohorts, including mortality $(0.05 \%$ vs $0.06 \%$, $p=0.4)$ and leak $(0.2 \%$ vs. $0.2 \%, p=0.3)$ rates.

\section{Discussion}

Sleeve gastrectomy has become the most commonly performed bariatric operation [5-7]. Due to this changing trend, there is heightened focus on short- and long-term outcomes following $\mathrm{SG}$, including strategies for minimizing complications. In this analysis of the 2015 and 2016 MBSAQIP-PUF database, we were able to reinforce the established safety of sleeve gastrectomy in the treatment of severe obesity [5, 18, 19, 21, 31]. Overall perioperative complication rates following SG remain very low. Staple line leak rate has been reported to be $1-3 \%$ in recent series [7, 19, 32-37] and is one of the most challenging postoperative complications to manage. SG leak may present acutely, early, intermediate, or as a late postoperative complication and often dictates which management course may be required to optimize outcome. Even though leak rates after SG have decrease over time $[32,35]$, it remains a clinically significant problem. It is the second most common cause of death following sleeve gastrectomy, with a reported associated mortality of $0.4 \%$ [38], prolonged hospitalization, and cost.

Surgeons have employed various intraoperative techniques (including the use of buttressing material, staple line oversewing, use of sealants, varying bougie sizes, and initiating sleeve at varying distances from the pylorus) to reduce the risk of a leak after SG, with varying outcomes [35, 36, 39-41]. Technical variables that may increase the risk for a leak may include ischemia, stenosis at the incisura, fundic redundancy, or use of an inappropriate staple height [42]. In addition to the technical variables, there are patient variables that may also 
Table 6 Outcomes following 1:1 case-control matching

\begin{tabular}{|c|c|c|c|}
\hline & $(+) \operatorname{SLR}[n=53,525]$ & $(-) \operatorname{SLR}[n=53,525]$ & $p$ value \\
\hline Postoperative LOS (days \pm sd) & $1.7 \pm 1.6$ & $1.5 \pm 1.3$ & $<0.0001$ \\
\hline \multicolumn{4}{|c|}{ 30-Day outcomes and perioperative complications, $n(\%)$} \\
\hline ICU admission & $229(0.4)$ & $241(0.5)$ & 0.6 \\
\hline Reoperation & $393(0.7)$ & $495(0.9)$ & 0.0006 \\
\hline Readmission & $1641(3.1)$ & $1601(3.0)$ & 0.5 \\
\hline Re-intervention & $468(0.9)$ & $479(0.9)$ & 0.7 \\
\hline Mortality & $35(0.07)$ & $32(0.06)$ & 0.7 \\
\hline Death related to operation & $21(0.04)$ & $15(0.03)$ & 0.3 \\
\hline Transfusion with $72 \mathrm{~h}$ & $231(0.4)$ & $291(0.5)$ & 0.009 \\
\hline Acute renal failure & $21(0.04)$ & $22(0.04)$ & 0.9 \\
\hline Progressive renal failure & $16(0.03)$ & $28(0.05)$ & 0.07 \\
\hline $\mathrm{CPR}$ & $9(0.02)$ & $13(0.02)$ & 0.4 \\
\hline Coma $>24 \mathrm{~h}$ & $3(0.01)$ & $3(0.01)$ & 1.0 \\
\hline Stroke & $7(0.01)$ & $3(0.01)$ & 0.2 \\
\hline Myocardial infarction & $11(0.02)$ & $15(0.03)$ & 0.4 \\
\hline On ventilator $>48 \mathrm{~h}$ & $26(0.05)$ & $22(0.04)$ & 0.5 \\
\hline Organ space SSI & $86(0.2)$ & $77(0.1)$ & 0.5 \\
\hline Deep incision SSI & $10(0.02)$ & $16(0.03)$ & 0.2 \\
\hline Superficial SSI & $109(0.2)$ & $123(0.2)$ & 0.4 \\
\hline Pneumonia & $65(0.1)$ & $73(0.1)$ & 0.5 \\
\hline Unplanned intubation & $43(0.08)$ & $52(0.1)$ & 0.4 \\
\hline DVT requiring therapy & $97(0.2)$ & $80(0.2)$ & 0.2 \\
\hline Pulmonary emboli & $50(0.09)$ & $40(0.07)$ & 0.3 \\
\hline Anticoagulation for DVT/PE & $208(0.4)$ & $204(0.4)$ & 0.8 \\
\hline Postoperative sepsis & $34(0.06)$ & $48(0.09)$ & 0.1 \\
\hline Postoperative septic shock & $15(0.03)$ & $12(0.02)$ & 0.6 \\
\hline Postoperative UTI & $134(0.3)$ & $135(0.3)$ & 1.0 \\
\hline \multicolumn{4}{|l|}{ Aggregate complications, $n(\%)$} \\
\hline Leak & $128(0.2)$ & $120(0.2)$ & 0.6 \\
\hline Bleeding & $117(0.2)$ & $155(0.3)$ & 0.02 \\
\hline Cardiovascular & $29(0.05)$ & $33(0.06)$ & 0.6 \\
\hline Pulmonary & $162(0.3)$ & $165(0.3)$ & 0.9 \\
\hline Renal & $54(0.1)$ & $42(0.08)$ & 0.2 \\
\hline VTE & $283(0.5)$ & $278(0.5)$ & 0.2 \\
\hline Surgical site infection & $231(0.4)$ & $244(0.5)$ & 0.6 \\
\hline Other Infection & $307(0.6)$ & $305(0.6)$ & 0.9 \\
\hline
\end{tabular}

SLR, staple line reinforcement; $n$, sample size; LOS, length of stay; sd, standard deviation; ICU, intensive care unit; CPR, cardiopulmonary resuscitation; SSI, surgical site infection; DVT, deep vein thrombosis; PE, pulmonary emboli; UTI, urinary tract infection; VTE, venous thromboembolic event

$P$ values that were statistically significant were bolded

increase the risk of a leak after sleeve gastrectomy that where not always accounted for in earlier studies. Patient-related variables that may increase the risk of a staple line leak following SG may include BMI, tobacco abuse, chronic use of steroid/ immunosuppression medications, poorer cardiopulmonary status, including oxygen dependence and sleep apnea, diabetes, hypertension, and poorer overall nutritional status [43].

The impact of staple line reinforcement utilization on leak rate following sleeve gastrectomy remains a point of controversy due to conflicting results in the published literature. In a systematic review by Gagner and Buchwald, they reported that the use of absorbable polymer member in stapling line reinforcement was projective of staple line leak, in comparison to no reinforcement, over-sewing, or staple line reinforcement with bovine pericardium [19]. In the first report from MBSAQIP, Berger et al. [5], using a logistical regression model, reported that SLR was associated with a higher staple line leak rate (9.96\% vs $0.65 \%$, OR 1.20$)$. Similar conflicting 
Table 7 Outcomes following case-control matching, stratified by operative duration

\begin{tabular}{|c|c|c|c|}
\hline & (+) SLR $[n=41,804]$ & $(-) \operatorname{SLR}[n=41,804]$ & $p$ value \\
\hline Postoperative LOS (days \pm sd) & $1.6 \pm 1.5$ & $1.5 \pm 1.3$ & $<0.0001$ \\
\hline \multicolumn{4}{|c|}{ 30-Day outcomes and perioperative complications, $n(\%)$} \\
\hline ICU admission & $138(0.3)$ & $174(0.4)$ & 0.04 \\
\hline Reoperation & $289(0.7)$ & $380(0.9)$ & 0.0004 \\
\hline Readmission & $1164(2.8)$ & $1198(2.9)$ & 0.5 \\
\hline Re-intervention & $324(0.8)$ & $365(0.9)$ & 0.1 \\
\hline Mortality & $19(0.05)$ & $25(0.06)$ & 0.4 \\
\hline Death related to operation & $10(0.02)$ & $14(0.03)$ & 0.4 \\
\hline Transfusion with $72 \mathrm{~h}$ & $151(0.4)$ & $225(0.5)$ & 0.0001 \\
\hline Acute renal failure & $9(0.02)$ & $19(0.05)$ & 0.06 \\
\hline Progressive renal failure & $19(0.05)$ & $11(0.03)$ & 0.1 \\
\hline CPR & $7(0.02)$ & $12(0.03)$ & 0.3 \\
\hline Coma $>24 \mathrm{~h}$ & $1(0)$ & $3(0.01)$ & 0.3 \\
\hline Stroke & $3(0.01)$ & $3(0.01)$ & 1.0 \\
\hline Myocardial infarction & $7(0.02)$ & $11(0.03)$ & 0.3 \\
\hline On ventilator $>48 \mathrm{~h}$ & $11(0.03)$ & $18(0.04)$ & 0.3 \\
\hline Organ space SSI & $66(0.2)$ & $51(0.1)$ & 0.2 \\
\hline Deep incision SSI & $8(0.02)$ & $14(0.03)$ & 0.2 \\
\hline Superficial SSI & $79(0.2)$ & $97(0.2)$ & 0.2 \\
\hline Pneumonia & $31(0.07)$ & $57(0.1)$ & 0.006 \\
\hline Unplanned intubation & $29(0.07)$ & $43(0.1)$ & 0.09 \\
\hline DVT requiring therapy & $60(0.1)$ & $68(0.2)$ & 0.5 \\
\hline Pulmonary emboli & $37(0.09)$ & $42(0.1)$ & 0.6 \\
\hline Anticoagulation for DVT/PE & $164(0.4)$ & $159(0.4)$ & 0.8 \\
\hline Postoperative sepsis & $26(0.06)$ & $37(0.09)$ & 0.2 \\
\hline Postoperative septic shock & $13(0.03)$ & $12(0.03)$ & 0.8 \\
\hline Postoperative UTI & $118(0.3)$ & $97(0.2)$ & 0.2 \\
\hline \multicolumn{4}{|l|}{ Aggregate complications, $n$ (\%) } \\
\hline Leak & $96(0.2)$ & $83(0.2)$ & 0.3 \\
\hline Bleeding & $79(0.2)$ & $119(0.3)$ & 0.004 \\
\hline Cardiovascular & $15(0.04)$ & $26(0.06)$ & 0.09 \\
\hline Pulmonary & $90(0.2)$ & $124(0.3)$ & 0.02 \\
\hline Renal & $30(0.07)$ & $31(0.07)$ & 0.9 \\
\hline VTE & $222(0.5)$ & $212(0.5)$ & 0.6 \\
\hline Surgical site infections & $164(0.4)$ & $184(0.4)$ & 0.3 \\
\hline Other infections & $232(0.6)$ & $222(0.5)$ & 0.6 \\
\hline All infections & $355(0.9)$ & $365(0.9)$ & 0.7 \\
\hline
\end{tabular}

SLR, staple line reinforcement; $n$, sample size; LOS, length of stay; sd, standard deviation; ICU, intensive care unit; CPR, cardiopulmonary resuscitation; SSI, surgical site infection; DVT, deep vein thrombosis; PE, pulmonary emboli; UTI, urinary tract infection; VTE, venous thromboembolic event $P$ values that were statistically significant were bolded

results have also been reported for SLR use in gastric bypass $[24,32]$. In a recent review of the Michigan Bariatric Surgery Collaborative, Varban et al. reported that after multivariate analysis, only the use of buttressing material was associated with a higher rate of leaks (OR 8.79; 95\% CI 2.49-31.01; $p=$ $0.0007)$, whereas the use of fibrin sealant was protective (OR $0.11 ; 95 \%$ CI $0.03-0.41 ; p=0.0013$ ) [32]. In a recent metaanalysis by Shikora and Mahoney [24] evaluating the advantages of SLR, they showed that leak rate in gastric bypass decreased progressively with the use of SLR, reporting leak rate of $2.60,2.44,1.88$, and $1 \%$ for no SRL, over-sewing staple line, reinforcement staple line with glycolide copolymer or bovine pericardium, respectively.

In our study, overall staple line leak rate following SG was $0.24 \%$, which is similar to that of other recent published series. In a review of the Michigan Bariatric Surgery Collaborative, 
Varban et al. [32] reported a leak rate of $0.36 \%$. In this study, there was no difference in the unmatched $(0.27 \%$ vs. $0.27 \%)$ and propensity matched $(0.3 \%$ vs. $0.3 \%$ ) cohort analysis. In our case-control matched analysis, in which cases and controls were statistically equally matched for all patient demographics and comorbidities, operative length, conversion, and initial surgical approach, the use of staple line reinforcement was neither protective nor deleterious $(0.2 \%$ vs $0.2 \%, p=0.6)$ in the development of a staple line leak. Our matched analysis accounts for much of the patient-related, as well as some of the technical confounders, that may impact sleeve leak rate and is an accurate reflection of the impact of SLR on leaks after SG. Leak after SG is likely multifactorial, and the lack of use of SLR is only one technical component that may have an impact on this outcome. Silecchia and Iossa highlighted this in their recent review, in which they outline technical features to be mindful of or consider in performing a sleeve gastrectomy in order to reduce the risk of a staple line leak. These include using a large bougie size, initiating sleeve 5-6 $\mathrm{cm}$ proximal to the pylorus, using an appropriate staple height, performing an appropriate staple line (avoiding corkscrewing of sleeve), avoiding narrowing of the incisura, removing crotch staples before deploying a subsequent staple, and reinforcing the staple line [25].

Similar to staple line leak, bleeding following sleeve gastrectomy is associated with increased morbidity, including a reoperation rate of $1.4 \%$ [25, 44], which is a $50-84 \%$ higher reoperation rate compared to that observed in our current analysis. Bleeding following SG is also multifactorial, as there are patient and technical variables that may increase the risk of postoperative staple line bleeding. Patient variables, such as hypertension, coagulopathy, super obesity, and obstructive sleep apnea, may increase the risk of bleeding $[45,46]$. In our matched analyses, bleeding was significantly higher in the cohort without staple line reinforcement. This is generally consistent with the published literature showing a protective effect of SLR use on bleeding after sleeve gastrectomy [5, 24]. Postoperative complications are often additive. In a review of the Michigan Bariatric Surgery Collaborative, Varban et al. [32] reported that leak rates were found to significantly correlate with transfusion requirement and conversion. As conversion and leak rates were similar in both our unmatched and matched cohort analysis, we were unable to draw any correlation between leak rates, transfusion requirement, and conversion.

The trend over the last several years has been towards increasing use of buttress material. Experts in the field have increasingly become more in favor of using some form of staple line reinforcement during sleeve gastrectomy. Between 2011 and 2014, those in favor of using buttressing increased from 77 to $81 \%$ [7]. This study reinforces the literature supporting the growing use of staple line reinforcement during sleeve gastrectomy. Its use is overall protective with reduced rates of bleeding, transfusion requirement, and reoperation, and no deleterious effect on the staple line leak.
There are several limitations to our study. First, this dataset does not provide relevant surgeon and surgical technique variables that may impact our primary outcome measures. Surgeon experience or case volume has been shown to be inversely correlated with bariatric surgery outcomes [47], including bleeding and leak rates. These are variables missing from the dataset and may add bias to our findings. The details about staple brand and staple loads used during sleeve were not available and may have impacted the outcomes reported. Secondly, even though this is a robust clinical dataset, there were some missing patient data. The percent of missing data points was very small and likely did not impact our analysis. Third, this is a retrospective analysis of prospectively collected data and is therefore vulnerable to biases associated with retrospective analysis of clinical dataset. Data entry is limited by the timeliness and completeness of data entry by the Bariatric Clinical Nurse Reviewers. Lastly, our study does not afford the ability to differentiate between the types of staple line reinforcement used. Prior studies have demonstrated outcome differences between the types of staple line reinforcement techniques and materials used [17, 19, 29]. While SLR is overall beneficial during sleeve, it is even more critical to fully understand the nuances of SLR techniques that will be most impactful in reducing these complications, including specific staple brands, height (colors), and SLR technique. Further investigation into these nuances, while adjusting for patient-related variables, will provide further insight and opportunities to minimize risks in the most commonly performed bariatric operation today.

\section{Conclusion}

In spite of the outlined limitations, the findings reported in this study and those reported by others show that using staple line reinforcement during sleeve gastrectomy is mostly protective of our bariatric patients. There is an appropriate increasing trend in the use of SLR during sleeve gastrectomy. Given the current data showing the protective effect of SLR use, there should be consideration for making the use of SLR a technical requirement during sleeve gastrectomy.

\section{Compliance with ethical standards}

Conflict of Interest None of the authors has a financial interest in any of the products, devices, or drugs mentioned in this manuscript.

Ethical Approval Statement This article does not contain any studies with human participants or animals performed by any of the authors. This is a retrospective review of a nationally available, clinical database containing only deidentified data. The dataset does not have any variables that would allow for patient, surgeon, or institution identification. For this type of study, neither an institutional review board approval nor formal consent was required. 


\section{Appendix}

Table 8 Aggregation of complications

\begin{tabular}{|c|c|}
\hline Aggregate variable & Composite variables \\
\hline Leak & $\begin{array}{l}\text { Reoperation with suspected reason: leak } \\
\text { Readmission with suspected reason: leak } \\
\text { Intervention with suspected reason: leak } \\
\text { Drain present over } 30 \text { days } \\
\text { Complication: organ space SSI }\end{array}$ \\
\hline Bleeding & $\begin{array}{l}\text { Reoperation with suspected reason: bleeding } \\
\text { Readmission with suspected reason: bleeding } \\
\text { Intervention with suspected reason: bleeding }\end{array}$ \\
\hline Cardiac/CVA & $\begin{array}{l}\text { Reoperation with suspected reason: cardiac NOS, CVA, or MI } \\
\text { Readmission with suspected reason: cardiac NOS, CVA, or MI } \\
\text { Intervention with suspected reason: cardiac NOS, CVA, or MI } \\
\text { Complication of CVA } \\
\text { Complication of MI }\end{array}$ \\
\hline Pulmonary & $\begin{array}{l}\text { Reoperation with suspected reason: shortness of breath, pneumonia, or other respiratory failure } \\
\text { Readmission with suspected reason: shortness of breath, pneumonia, or other respiratory failure } \\
\text { Intervention with suspected reason: shortness of breath, pneumonia, or other respiratory failure } \\
\text { Complication: on ventilator }>48 \mathrm{~h} \\
\text { Complication: unplanned intubation } \\
\text { Complication: pneumonia }\end{array}$ \\
\hline Renal & $\begin{array}{l}\text { Reoperation with suspected reason: renal insufficiency } \\
\text { Readmission with suspected reason: renal insufficiency } \\
\text { Intervention with suspected reason: renal insufficiency } \\
\text { Complication: progressive renal insufficiency } \\
\text { Complication: acute renal failure }\end{array}$ \\
\hline DVT or PE & $\begin{array}{l}\text { Reoperation with suspected reason: vein thrombosis requiring therapy or pulmonary embolism } \\
\text { Readmission with suspected reason: vein thrombosis requiring therapy or pulmonary embolism } \\
\text { Intervention with suspected reason: vein thrombosis requiring therapy or pulmonary embolism } \\
\text { Complication: vein thrombosis requiring therapy } \\
\text { Complication: pulmonary embolism } \\
\text { Complication: anticoagulation initiated of presumed/confirmed vein thrombosis/PE }\end{array}$ \\
\hline Wound infection & $\begin{array}{l}\text { Reoperation with suspected reason: wound infection or other abdominal sepsis } \\
\text { Readmission with suspected reason: wound infection or other abdominal sepsis } \\
\text { Intervention with suspected reason: wound infection or other abdominal sepsis } \\
\text { Complication: postop superficial incisional SSI occurrence } \\
\text { Complication: postop deep incisional SSI occurrence }\end{array}$ \\
\hline Other infection & $\begin{array}{l}\text { Reoperation with suspected reason: infection/fever } \\
\text { Readmission with suspected reason: infection/fever } \\
\text { Intervention with suspected reason: infection/fever } \\
\text { Complication: postop sepsis occurrence } \\
\text { Complication: postop septic shock occurrence } \\
\text { Complication: postop pneumonia occurrence } \\
\text { Complication: postop urinary tract infection occurrence }\end{array}$ \\
\hline Total infection & $\begin{array}{l}\text { Wound infection, as above } \\
\text { Other infection, as above }\end{array}$ \\
\hline
\end{tabular}

SSI, surgical site infection; CVA, cardiovascular accident; NOS, not otherwise specified; MI, myocardial infarction; DVT, deep vein thrombosis; PE, pulmonary emboli 


\section{References}

1. WHO. Obesity and overweight. WHO. www.who.int/en/newsroom/fact-sheets/detail/obesity-and-overweight. Published 2018, Accessed August 21, 2018

2. Peterli R, Wölnerhanssen BK, Peters T, et al. Effect of laparoscopic sleeve gastrectomy vs laparoscopic Roux-en-Y gastric bypass on weight loss in patients with morbid obesity: the SM-BOSS randomized clinical trial. JAMA. 2018;319(3):255-65.

3. Salminen P, Helmiö M, Ovaska J, et al. Effect of laparoscopic sleeve gastrectomy vs laparoscopic Roux-en-Y gastric bypass on weight loss at 5 years among patients with morbid obesity: the SLEEVEPASS randomized clinical trial. JAMA. 2018;319(3): 241-54.

4. Kang JH, Le QAJM. Effectiveness of bariatric surgical procedures: a systematic review and network meta-analysis of randomized controlled trials. Medicine (Baltimore). 2017;96(46):e8632.

5. Berger ER, Clements RH, Morton JM, et al. The impact of different surgical techniques on outcomes in laparoscopic sleeve gastrectomies: the first report from the Metabolic and Bariatric Surgery Accreditation and Quality Improvement Program (MBSAQIP). Ann Surg. 2016;264(3):464-73.

6. Abraham A, Ikramuddin S, Jahansouz C, et al. Trends in bariatric surgery: procedure selection, revisional surgeries, and readmissions. Obes Surg. 2016;26(7):1371-7.

7. Varela JE, Nguyen NT. Laparoscopic sleeve gastrectomy leads the U.S. utilization of bariatric surgery at academic medical centers. Surg Obes Relat Dis. 2015;11(5):987-90.

8. Durmush EK, Ermerak G, Durmush D. Short-term outcomes of sleeve gastrectomy for morbid obesity: does staple line reinforcement matter? Obes Surg. 2014;24(7):1109-16.

9. Gagner M, Hutchinson C, Rosenthal R. Fifth International Consensus Conference: current status of sleeve gastrectomy. Surg Obes Relat Dis. 2016;12(4):750-6.

10. Giannopoulos GA, Tzanakis NE, Rallis GE, et al. Staple line reinforcement in laparoscopic bariatric surgery: does it actually make a difference? A systematic review and meta-analysis. Surg Endosc. 2010;24(11):2782-8.

11. Hoyuela C. Five-year outcomes of laparoscopic sleeve gastrectomy as a primary procedure for morbid obesity: a prospective study. World J Gastrointest Surg. 2017;9(4):109-17.

12. Knapps J, Ghanem M, Clements J, et al. A systematic review of staple-line reinforcement in laparoscopic sleeve gastrectomy. JSLS. 2013;17(3):390-9.

13. Lee JH, Nguyen Q-N, Le QA. Comparative effectiveness of 3 bariatric surgery procedures: Roux-en-Y gastric bypass, laparoscopic adjustable gastric band, and sleeve gastrectomy. Surg Obes Relat Dis. 2016;12(5):997-1002.

14. Maciejewski ML, Arterburn DE, Van Scoyoc L, et al. Bariatric surgery and long-term durability of weight loss. JAMA Surg. 2016;151(11):1046-55

15. Nassour I, Almandoz JP, Adams-Huet B, et al. Metabolic syndrome remission after Roux-en-Y gastric bypass or sleeve gastrectomy. Diabetes, metabolic syndrome and obesity: targets and therapy. Diabetes Metab Syndr Obes. 2017;10:393-402.

16. DuPree CE, Blair K, Steele SR, et al. Laparoscopic sleeve gastrectomy in patients with preexisting gastroesophageal reflux disease: a national analysis. JAMA Surg. 2014;149(4):328-34.

17. Inaba CS, Koh CY, Sujatha-Bhaskar S, et al. One-year mortality after contemporary laparoscopic bariatric surgery: an analysis of the bariatric outcomes longitudinal database. J Am Coll Surg. 2018;226(6):1166-74.

18. Hutter MM, Schirmer BD, Jones DB, et al. First report from the American College of Surgeons-Bariatric Surgery Center Network: laparoscopic sleeve gastrectomy has morbidity and effectiveness positioned between the band and the bypass. Ann Surg. 2011;254(3):410-22.

19. Gagner M, Buchwald JN. Comparison of laparoscopic sleeve gastrectomy leak rates in four staple-line reinforcement options: a systematic review. Surg Obes Relat Dis. 2014;10(4):713-23.

20. Khoursheed M, Al-Bader I, Mouzannar A, et al. Postoperative bleeding and leakage after sleeve gastrectomy: a single-center experience. Obes Surg. 2016;26(12):2944-51.

21. Wang Z, Dai X, Xie H, et al. The efficacy of staple line reinforcement during laparoscopic sleeve gastrectomy: a meta-analysis of randomized controlled trials. Int J Surg. 2016;25:145-52.

22. Nielsen AW, Helm MC, Kindel T, et al. Perioperative bleeding and blood transfusion are major risk factors for venous thromboembolism following bariatric surgery. Surg Endosc. 2018;32(5):248895

23. Dick A, Byrne TK, Baker M, et al. Gastrointestinal bleeding after gastric bypass surgery: nuisance or catastrophe? Surg Obes Relat Dis. 2010;6(6):643-7.

24. Shikora SA, Mahoney CBJO. Clinical benefit of gastric staple line reinforcement (SLR) in gastrointestinal surgery: a meta-analysis. Obes Surg. 2015;25(7):1133-41.

25. Silecchia G, Iossa A. Complications of staple line and anastomoses following laparoscopic bariatric surgery. Ann Gastroenterol. 2018;31(1):56-64.

26. Rached AA, Basile M, El Masri H. Gastric leaks post sleeve gastrectomy: review of its prevention and management. World J Gastroenterol. 2014;20(38):13904-10.

27. Warner DL, Sasse KC. Technical details of laparoscopic sleeve gastrectomy leading to lowered leak rate: discussion of 1070 consecutive cases. Minim Invasive Surg. 2017;2017

28. Khuri SF, Henderson WG, Daley J, et al. The patient safety in surgery study: background, study design, and patient populations. J Am Coll Surg. 2007;204(6):1089-102.

29. Cohen ME, Ko CY, Bilimoria KY, et al. Optimizing ACS NSQIP modeling for evaluation of surgical quality and risk: patient risk adjustment, procedure mix adjustment, shrinkage adjustment, and surgical focus. J Am Coll Surg. 2013;217(2):336-46. e331

30. Ingraham AM, Richards KE, Hall BL, et al. Quality improvement in surgery: the American College of Surgeons national surgical quality improvement program approach. Adv Surg. 2010;44(1): 251-67.

31. Chang SH, Stoll CR, Song J, et al. The effectiveness and risks of bariatric surgery: an updated systematic review and meta-analysis, 2003-2012. JAMA Surg. 2014;149(3):275-87.

32. Varban OA, Sheetz KH, Cassidy RB, et al. Evaluating the effect of operative technique on leak rates after laparascopic sleeve gastrectomy: a case control study. Surg Obes Relat Dis. 2017;13:560-7.

33. Angrisani L, Cutolo PP, Buchwald JN, et al. Laparoscopic reinforced sleeve gastrectomy: early results and complications. Obes Surg. 2011;21(6):783-93.

34. Aurora AR, Khaitan L, Saber AA. Sleeve gastrectomy and the risk of leak: a systematic analysis of 4,888 patients. Surg Endosc. 2012;26(6):1509-15.

35. Gagner M. Decreased incidence of leaks after sleeve gastrectomy and improved treatments. Surg Obes Relat Dis. 2014;10(4):611-2.

36. Parikh M, Issa R, McCrillis A, et al. Surgical strategies that may decrease leak after laparoscopic sleeve gastrectomy: a systematic review and meta-analysis of 9991 cases. Ann Surg. 2013;257(2): 231-7.

37. Sakran N, Goitein D, Raziel A, et al. Gastric leaks after sleeve gastrectomy: a multicenter experience with 2,834 patients. Surg Endosc. 2013;27(1):240-5.

38. Jurowich C, Thalheimer A, Seyfried F, et al. Gastric leakage after sleeve gastrectomy - clinical presentation and therapeutic options. Langenbeck's Arch Surg. 2011;396:981-7. 
39. Yuval JB, Mintz Y, Cohen MJ, et al. The effects of bougie caliber on leaks and excess weight loss following laparoscopic sleeve gastrectomy. Is there an ideal bougie size. Obes Surg. 2013;23(10):168591.

40. Musella M, Milone M, Maietta P, et al. Laparoscopic sleeve gastrectomy: efficacy of fibrin sealant in reducing postoperative bleeding. A randomized controlled trial. Updat Surg. 2014;66(3):197201.

41. D'Ugo S, Gentileschi P, Benavoli D, et al. Comparative use of different techniques for leak and bleeding prevention during laparoscopic sleeve gastrectomy: a multicenter study. Surg Obes Relat Dis. 2014;10(3):450-4.

42. Iossa A, Abdelgawad M, Watkins MB, et al. Leaks after laparoscopic sleeve gastrectomy: an over of pathogenesis and risk factors. Langenbeck's Arch Surg. 2016;401(6):757-66.

43. Reza FA, Li S, Inaba C, et al. Risk factors for gastrointestinal leak after bariatric surgery: MBSAQIP analysis. J Am Coll Surg. 2018;227(1):135-41.
44. Gagner M, Deitel M, Erickson AL, et al. Survey on laparoscopic sleeve gastrectomy (LSG) at the Fourth International Consensus Summit on Sleeve Gastrectomy. Obes Surg. 2013;23:2013-7.

45. Janik MR, Walędziak M, Bragoszewski J, et al. Prediction model for hemorrhagic complications after laparoscopic sleeve gastrectomy: development of SLEEVE BLEED calculator. Obes Surg. 2017;27:968-72.

46. de Angelis F, Abdelgawad M, Rizzello M, et al. Perioperative hemorrhagic complications after laparoscopic sleeve gastrectomy: fouryear experience of a bariatric center of excellence. Surg Endosc. 2017;31:3547-51.

47. Noel P, Nedelcu M, Gagner M. Impact of the surgical experience on leak rate after laparoscopic sleeve gastrectomy. Obes Surg. 2016;26:1782-7.

Publisher's NoteSpringer Nature remains neutral with regard to jurisdictional claims in published maps and institutional affiliations. 